\title{
Comprehensive assessment of health risks from cars, including photochemical oxidants and noises
}

\author{
Y. OKAZAKI ${ }_{1}^{*}$, L. ITO ${ }_{1}$, A. TOKAI 1
}

1 Graduate School of Engineering, Osaka University, 2-1

Yamadaoka, Suita, Osaka 565-0871, Japan

(*correspondence : okazaki@em.see.eng.osaka-u.ac.jp)

The expansion of car traffic has resulted in serious pollution such as air pollution and noise. The nitrogen oxides (NOx) emitted from cars react with sunlight in the atmosphere to produce photochemical oxidants, which causes respiratory symptoms, pulmonary function changes, and so on [1]. However, few studies comprehensively assess such health risks from cars. In this study, the distribution of atmospheric concentrations of $\mathrm{NO}_{\mathrm{x}}$ and photochemical oxidants was estimated, and relevant health risks were also evaluated. In addition, we estimated the health risks of road traffic noise, which causes ischemic heart disease and high sleep disturbance [2]. Finally, we assessed each risk using the same index, DALYs (Disability-Adjusted Life Years).

The roadsides of Osaka City in Japan were selected as the target area, which is also the target of road traffic census. ADMER-PRO ver. 1.0, a model for the estimation of concentrations of secondary products, was used to predict the distribution of $\mathrm{NO}_{2}$ and $\mathrm{O}_{3}$. The health risks of air pollutants were evaluated from the concentrations, using the LCIA (Life Cycle Impact Assessment) method. The ASJ RTN-Model 2013 [3] was used to predict road traffic noise. In the health risk analysis, we set the assumption that ischemic heart disease and high sleep disturbance were caused by the noise generated in daytime and night-time, respectively $[4,5]$.

The results of this study showed that the health risks of road traffic noise outweighed those of air pollutants. Our results will be useful for transportation policies in the future.

[1] WHO Europe (2000). [2] WHO Europe (2011). [3] The Research Committee on Road Traffic Noise in the Acoustical Society of Japan (2014). [4] Babisch W (2008). [5] Miedema HME, et al. (2003). 